prevention interventions should focus on condom use among this group.

Disclosure No significant relationships.

\subsection{EPIDEMIOLOGICAL IMPACT OF EXPEDITED PARTNER THERAPY FOR MEN WHO HAVE SEX WITH MEN: A MODELING STUDY}

${ }^{1}$ Kevin Weiss*, ${ }^{1}$ Jeb Jones, ${ }^{2}$ David Katz, ${ }^{3}$ Thomas Gift, ${ }^{3}$ Kyle Bernstein, ${ }^{3}$ Kimberly Workowski, ${ }^{4}$ Eli Rosenberg, 'Samuel Jenness. 'Emory University, Epidemiology, Atlanta, USA; ' University of Washington, Global Health, Seattle, USA; ${ }^{3}$ Centers for Disease Control and Prevention, Division of STD Prevention, Atlanta, USA; ${ }^{4}$ SUNY - University at Albany, Epidemiology and Biostatistics, Albany, USA

10.1136/sextrans-2019-sti.172

Background Expedited partner therapy (EPT) is an intervention for patients with gonorrhea (NG) or chlamydia (CT), through which index patients are provided with medication to give to their partner. While EPT is recommended for heterosexuals, EPT is not recommended for men who have sex with men (MSM), partially due to concerns about overtreatment of uninfected partners and missed opportunities for HIV diagnosis. In this study, we used modeling to investigate the potential impact of EPT implementation on STI incidence among MSM in the United States.

Methods We extended our stochastic network-based mathematical model of HIV, NG, and CT among MSM to include partner-delivered EPT for NG and CT. EPT implementation was simulated for 10 years. Counterfactual scenarios varied EPT coverage, provision, uptake, and partnership window duration. We estimated STI incidence, percent of infections averted (PIA), and process outcomes under each scenario, compared to a reference scenario that included routine and symptomsbased STI screening at current empirical levels among US MSM.

Results Delivery of EPT to 20\% of eligible MSM index patients (coverage) was projected to reduce cumulative STI infections among MSM by $22 \%$ over 10 years compared to current estimated STI screening levels. A $20 \%$ increase in providing medication to non-index partners (provision) averted $29 \%$ of STI infections compared to STI screening. By partnership type, intervention benefits were greatest when EPT was restricted to casual partners. The proportion of partners given medication who had no current STI varied from $55 \%$ to $65 \%$, depending on coverage. The proportion of partners given medication with undiagnosed HIV infection was $4 \%$.

Conclusion EPT could substantially reduce bacterial STI incidence for MSM. However, this intervention could result in a substantial increase in unnecessary use of antibiotic medication by STI-uninfected MSM, raising concerns about cost and antimicrobial resistance in absence of additional medical evaluation.

Disclosure No significant relationships.

\section{4 \\ EFFECTS OF ENHANCED STI PARTNER NOTIFICATION COUNSELING IN CAPE TOWN: RANDOMIZED CONTROLLED TRIAL}

${ }^{1}$ Catherine Mathews ${ }^{*},{ }^{2}$ Moira Kalichman, ${ }^{1}$ Sarah Dewing, ${ }^{2}$ Ellen Banas, ${ }^{1}$ Sekelwa Dumile, ${ }^{1}$ Amanda Mdlikiva, ${ }^{1}$ Thembinkosi Mdlikiva, ${ }^{3}$ Karen Jennings, ${ }^{4}$ Mark Lurie, ${ }^{2}$ Seth Kalichman. 'South African Medical Research Council, Health Systems Research Unit, Cape Town, South Africa; ${ }^{2}$ University of Connecticut, Department of Psychology, Storrs, USA; ${ }^{3}$ City of Cape Town, Department Of Health, Cape Town, South Africa; ${ }^{4}$ Brown University, School of Public Health, Providence, USA

\subsection{6/sextrans-2019-sti.173}

Background People who contract STI in high-HIV prevalence settings such as South Africa are among the highest risk populations for HIV. Single-session behavioural interventions can reduce a person's risk for STI with added HIV prevention benefits, but promising prevention effects are undermined by untested, untreated partners with persistent STI. We tested a theory-based, single session behavioural risk reduction intervention with enhanced partner notification (ePN) counselling among people diagnosed with an STI, in Cape Town, South Africa.

Methods Participants were 1050 consenting adults diagnosed with STI at a community clinic. After the standard STI/HIV consultation and treatment, participants were randomly allocated to: (a) 20 minutes health education; (b) 45 minutes risk reduction skills counseling; or (c) 45 minutes risk reduction and ePN communication skills counseling. We measured participants' reports of PN to potentially exposed partners two weeks after diagnosis.

Results Participants were 527 men and 523 women, 20\% HIV positive, enrolled between 2014 and 2017. There were no significant baseline differences between trial arms in number of reported main or casual sex partners. Two weeks after STI diagnosis ( $n=890,85 \%$ retained), $77 \%$ of participants had notified a main partner of their STI and 57\% had notified a casual partner. Conditions did not differ in rates of notifying main partners. However, participants receiving the ePN counseling $(57 \%)$ were significantly more likely to have notified a casual partner compared to those in the health education (41\%) and the risk reduction counseling (41\%) arms, OR = $1.87, \mathrm{p}<0.05$. This pattern of differences did not differ by gender, with intervention effects significant for both men and women.

Conclusion The ePN intervention was effective at improving PN with casual partners. Although partner linkage to care was not measured, future analyses will determine whether there was a reduction in repeat STIs in index cases.

Disclosure No significant relationships.

\subsection{PARTNER NOTIFICATION OUTCOMES FOR CHLAMYDIA/ GONORRHEA CASES DIAGNOSED THROUGH CLINICS VS GETCHECKEDONLINE IN BRITISH COLUMBIA}

${ }^{1}$ Arina Zamanpour*, ${ }^{1}$ Jason Wong, ${ }^{1}$ Devon Haag, ${ }^{1}$ Troy Grennan, ${ }^{2}$ Dee Hoyano, ${ }^{2}$ Sophie Bannar-Martin, ${ }^{3}$ Silvina Mema, ${ }^{3}$ Maja Karlsson, ${ }^{4}$ Christopher Fairley, ${ }^{1}$ Gina Ogilvie, ${ }^{1}$ Mark Gilbert. 'British Columbia Centre for Disease Control, Vancouver, Canada; ${ }^{2}$ sland Health Authority, Victoria, Canada; 3 Interior Health Authority, Kelowna, Canada; ${ }^{4}$ Melbourne Sexual Health Centre, Melbourne, Australia

10.1136/sextrans-2019-sti.174

Background GetCheckedOnline (GCO) is an internet-based testing service for sexually transmitted and blood-borne 
infections in British Columbia (BC), Canada that is highly accepted by clients. The literature has not addressed whether treatment and partner notification (PN) outcomes differ between clients of internet-based testing services and clinicbased clients. We sought to compare treatment uptake, engagement in PN, and PN outcomes between chlamydia (CT) and/ or gonorrhea (GC) cases diagnosed through GCO and two provincially-operated sexually transmitted infection clinics.

Methods A matched case-control study was conducted among CT/GC cases in BC from 2016-2018. All cases diagnosed through GCO were selected and matched to two clinic cases (controls) based on diagnosis, gender, age group, and specimen collection date. Using a cascade of care, PN outcomes were compared between partners of GCO and clinic cases. Bivariate comparisons were conducted using chi-square or Fisher's exact test.

Results There were 257 GCO cases matched to 514 clinic cases. Treatment uptake did not differ between GCO (254/ $257,98.9 \%)$ and clinic $(513 / 514,99.8 \%)$ cases. There was no difference in the proportion of notified partners between GCO (176/287, 61.3\%) and clinic cases (338/520, 65.0\%) although a greater proportion $(\mathrm{P}<0.01)$ of notifiable partners reported by clinic cases were notified by public health (44/ $520,8.5 \%)$ vs. GCO cases $(6 / 287,2.1 \%)$. Among all notified partners, clinic cases reported a greater proportion $(\mathrm{P}<0.01)$ of tested partners $(114 / 338,33.7 \%)$ than did GCO cases (38/ $176,21.6 \%)$. Of all notifiable partners, positivity was higher $(\mathrm{P}<0.01)$ among clinic cases $(93 / 520,17.9 \%)$ than GCO cases (29/287, 10.1\%).

Conclusion GCO clients diagnosed with CT or GC demonstrated similar treatment uptake and engagement in $\mathrm{PN}$ to clinic clients. The difference in partner testing may be due to different populations being reached by internet-based testing and merits further investigation. The high positivity rate among partners underscores the importance of PN.

Disclosure No significant relationships.

\subsection{CONTACT TRACING VS PATIENT-DELIVERED PARTNER TREATMENT FOR AFRICAN AMERICAN HETEROSEXUAL MEN WITH CHLAMYDIA}

\begin{abstract}
${ }^{1}$ Patricia Kissinger*, ${ }^{2}$ Norine Schmidt, ${ }^{2}$ Gérard Gomes, ${ }^{2}$ Glenis Scott, ${ }^{2}$ Shannon Watson, ${ }^{2}$ Megan Clare Craig-Kuhn, ${ }^{3}$ Phazal Hines, ${ }^{3}$ Sean Clark, ${ }^{3}$ Javone Davis, ${ }^{4}$ Alyssa Lederer, ${ }^{2}$ David Martin. ${ }^{1}$ Tulane School of Public Health and Tropical Medicine, New Orleans, USA; ${ }^{2}$ Tulane University School of Public Health and Tropical Medicine, Epidemiology, New Orleans, USA; ${ }^{3}$ Louisiana Office of Public Health, HIVISTI, New Orleans, USA; ${ }^{4}$ Tulane University School of Public Health and Tropical Medicine, Global Community Health and Behavior, New Orleans, USA
\end{abstract}

\subsection{6/sextrans-2019-sti.175}

Background Most research on partner treatment for chlamydia has been done with female index cases or with clinic-based populations, thus less is known about optimal approaches for community screened men. The purpose of this study is to compare contact-tracing versus patient-delivered partner-treatment (PDPT) for rates of index and partner treatment among chlamydia infected-young heterosexual African American men (AA) diagnosed by screening at community venues.

Methods 'Check It' is a community chlamydia screening program for AA men aged 15-24. Initially, index and partner treatment was done by a disease intervention specialist (DIS) using a contact-tracing approach where contacted index and partners could get azithromycin treatment at no cost at participating pharmacies. In month 14 of the program, in response to the findings of in-depth interviews with men enrolled in the program, contacted index men were offered PDPT that could be picked up at a participating pharmacy or mailed to the index. Index and partner treatment outcomes were compared.

Results In-depth interviews revealed concerns about giving DIS contact partner information and issues with transportation, thus, PDPT and direct-mailing of azithromycin were added to treatment options. There were 102 screened men in the contact-tracing-phase and 22 in the PDPT-phase. Of these $124 \mathrm{Ct}$ + men, $83.9 \%$ were contacted and $62.9 \%$ were treated and they reported 204 baseline partners. Treatment rates were higher in the PDPT-phase versus the contact-tracing-phase for index $(86.4 \%$ vs $57.8 \%$, p-value $<0.02)$ and partners $(50.0 \%$ vs $20.6 \%$, p-value $<0.01)$ respectively. Time-to-treatment was shorter for those in the PDPT-phase versus the contact-tracing-phase for index (10.7 vs 16.8 days, $\mathrm{p}<0.04)$. During the PDPT-phase 8/22 index men (36.4\%) opted for mail-delivery of azithromycin.

Conclusion Expedited index and PDPT using local pharmacies or mailed-treatment resulted in significantly higher index and partner treatment rates and shorter time-to-treatment among AA young men screened for $\mathrm{Ct}$ in the community.

Disclosure No significant relationships.

\section{3 - WORKING TOWARDS SEXUAL HEALTH EQUITY: UNDERSTANDING CONTEXT}

\section{Wednesday, July 17, 2019 10.45 AM - 12:15 PM}

\subsection{FACTORS ASSOCIATED WITH SAFER SEX EFFICACY AMONG NORTHERN AND INDIGENOUS YOUTH IN THE NORTHWEST TERRITORIES, CANADA}

${ }^{1}$ Carmen Logie*, ${ }^{2}$ Candice Lys, ${ }^{2}$ Kayley Mackay, ${ }^{2}$ Nancy Macneill, ${ }^{1}$ Analaura Pauchulo, ${ }^{3}$ Abdool Yasseen. 'University of Toronto, Factor-inwentash Faculty of Social Work, Toronto, Canada; ${ }^{2}$ Fostering Open eXpression Among Youth, Yellowknife, Canada; ${ }^{3}$ University of Toronto, Dalla Lana School of Public Health, Toronto, Canada

\subsection{6/sextrans-2019-sti.176}

Background Identifying social and structural factors associated with sexually transmitted infections (STI) vulnerability is urgent in the Northwest Territories (NWT), where STI prevalence is 7-fold the Canadian average. The NWT also experiences higher food insecurity and intimate partner violence (IPV) than the national average. Safer sex efficacy (SSE) comprises knowledge, intention, and relationship dynamics for safer sex negotiation. We examined social and structural factors associated with SSE among Northern and Indigenous adolescents in the NWT.

Methods With an Indigenous sexual health agency, we conducted a cross-sectional survey with adolescents aged 13-17 in 17 NWT communities. Summary statistics and statistical comparisons were conducted, followed by crude and multivariable regression models, with a canonical link function, to compare factors associated with SSE and within gender stratifications. We conducted post-hoc sensitivity analyses among Indigenous youth. 